\title{
TIETZE'S SYNDROME - A CASE OF DISTURBED BIOMECHANICS OR A REAL ENIGMA?
}

\author{
Todorov T. ${ }^{1}$, E. Ilieva $^{2}$, I. Todorov ${ }^{1}$ \\ ${ }^{1}$ Clinic of Physiotherapy, Rehabilitation and Thalassotherapy, St. Marina University Hospital of \\ Varna, Medical University of Varna; ${ }^{2}$ Department of Physical and Rehabilitation Medicine, \\ Medical University of Plovdiv
}

\begin{abstract}
Tietze's syndrome continues to confuse the clinicians although it has been first reported in 1921. A major reason for that is the controversial information about its unclear etiology and pathogenesis. Very often, the clinical findings resemble other diseases and the conventional drug therapy is ineffective. All these facts may turn the diagnosis and treatment of Tietze's syndrome into a real challenge. The information about this syndrome available has been systematized. A special attention has been paid to the etiology, diagnosis and modern therapeutic modalities.
\end{abstract}

Key words: Tietze's syndrome, etiology, clinical symptoms, biomechanics, manual diagnosis, treatment

\section{INTRODUCTION}

Tietze's syndrome has been officially reported for a first time in 1921 by the German surgeon Alexander Tietze (1864-1927). He described this syndrome in the journal 'Berliner klinische Wochenschrift' in the following way: "It is a matter of painful swelling of the upper rib cartilage of the left side of the chest observed by 3-4 patients at the age between 18 and 50. The pathology has developed for several weeks/months and has shown an improvement with time" (13).

\section{Clinical description of the syndrome}

This syndrome presents with an obvious pathology. However, at the same time, its etiology remains unknown, which often confuses the clinicians. Its variability contributes to this confusion. The symptoms of the syndrome could be present for a week, month or ever some years. It is possible that they suddenly disappear and then, after a variable remission, they relapse again.

In the literature available, there is contradictory evidence of this disease, defining it as a self restricted one and patient's worsening for about a month. Unanimity exists only about the localization:

1. The actual anatomic localization of the lesion is the costosternal cartilage of the ribs I, II, or III.

2. The lesion in the cartilage of rib II is considered a classical finding.

\footnotetext{
Address for correspondence

I. Todorov, Clinic of Physiotherapy, Rehabilitation and Thalassotherapy, St. Marina University Hospital of Varna,

Medical University of Varna

55 Marin Drinov Str., 9002 Varna, Bulgaria

e-mail: ilkotodorov@gmail.com
}

The actual findings are swelling and pain. More commonly, they are left-sided (5). The pain could emerge suddenly, to be quite severe and even to simulate a stenocardiac attack. It could get stronger if pressing the sternum or the neighbouring ribs $(3,14)$. Some patients report a stabbing pain and shortness of breath (5).

Costochondritis characterized by painful, tender but non-swollen chondrosternal joints being the more common disease is reported as a differential diagnosis for Tietze's syndrome (4)

\section{Etiology}

Because the syndrome develops quite similarly to much more threatening diseases, at the first stage, referred pain from the heart and fractures should be excluded.

Several different reasons for the syndrome have been mentioned in the literature:

1. Previous ossification and calcification of the rib cartilage

2. Congenital fork-shaped deformation of the ribs

3. Hormonal disorder

4. Allergy

5. Difference in the lower limbs length causing a chronic posture imbalance

6. Rheumatoid arthritis

7. Pregnancy

8. Hodgkin's disease

9. Diabetes mellitus

10. Haemochromatosis

11. Cirrhosis

12. Aseptic necrosis

In most cases, other pathological findings are impossible to be found. In about $80 \%$ of the cases reported the pain is be- 
The Tietze syndrome - a case of disturbed biomechanics or a real enigma?

nign, of which musculoskeletal chest pain accounts for almost 50\% (12).

Tietze's syndrome could be triggered at any age, but, most probably, in the 4th decade of life (3).

It was impossible to reveal any evident pathogenesis for the pain and swelling in Tietze's syndrome and that is why the trauma and strong persistent cough were considered an important reason for this syndrome (2). A mechanical acute costochondritis induced by cough of unknown origin was suspected, too (6). The inflammation of the chondrosternal joints caused, probably, by repetitive straining of muscles attaching to involved ribs or a costotransverse joint dysfunction was suspected, too $(1,10)$.

Tietze's syndrome should be considered in those patients who present with severe breast pain several months after reconstructive breast surgery (15).

Some cases could be related with viral infections and pathological rib configurations. In most of them, the pathogenesis is unclear and confusing. Some authors describe pain irradiation towards the arm of the same side alongside with paresthesia and weakness. There are many reported cases, where women experiencing hard physical labour are involved, with chronic traumatizing the upper costal cartilage $(3,14)$.

\section{Pathology}

The pathological findings have contributed a very little to the clarification of Tietze's syndrome. Most often, the pathologists use vague definitions such as 'dystrophy' and 'an increased vascularization, mucoid findings, and perichondral inflammation'.

The laboratory parameters deliver almost no information at all. The nuclear diagnostics and contrast matter examinations exclude pathological processes similar to morbus Bechterew, cellulitis, and morbus Reiter.

The scintigraphy with technetium shows a reaction of the painful zones, however, the results refer to a small groups of patients and are lacking scientific significance.

The x-ray data are based mainly on excluding the other diseases. According to some authors, the computer tomography doesn't visualize any obvious changes (11). In another study (16) MRI demonstrates an enlargement and thickening of the cartilage at the side of complaint along with increased signal intensities of affected cartilage (in all the 12 patients) as well as a bone marrow oedema (in 5 of 12 patients). Besides there is a vivid gadolinium uptake in the areas of thickened cartilage (in 10 of 12 patients), in the subchondral bone marrow (in 4 of 12 patients) and/or in the capsule and ligaments (in 7 of 12 patients).

No infectious or chemical influence could be proved (9). No specific alterations cold be established by using the electron microscopy, x-ray and radioisotopes as well (9).

Based on all these reasons listed above, an obvious question emerges, whether certain biomechanical factors and imbalance issues are involved and if they could play a certain role in the provocation, development and the reversal of Tietze's syndrome. Could, eventually, a scoliotic imbal- ance lead to a mechanical deviation and traumatizing the rib II cartilage and thus initiate Tietze's syndrome?

\section{Manual diagnosis}

Many investigations have confirmed that the major finding of Tietze's syndrome is the extension of the corresponding segment of movement $(3,7,8)$. Pain is established during movement or by manually palpating the tissues in the projection of the intervertebral joints and the transverse processes. In the corresponding vertebral segment, a relatively free extension and a limited flexion is reported during palpation. The limitation of intervertebral joints' movement could be accompanied by limitation of the associated rib movement in most cases. The relative frontal protrusion of the vertebral body in comparison with the bodies of the upper and lower vertebrae leads to a limited ventral movement of either one of the transverse processes, or of both of them. These changes cause a ventral reposition of the corresponding rib. Ventralizing the vertebra as explained in this way clarifies the frontal deviation, rounding and swallowing of the costosternal cartilage. An additional scoliotic lateral bending could cause a rotation of the vertebra that would increase further rib frontal protrusion and deviation. All these reasons deliver an acceptable mechanism for the pathogenesis of Tietze's syndrome. However, they do not explain the predilection for the involvement of the costosternal cartilage of the $2^{\text {nd }}$ rib at all.

Because the above mentioned reasons it is necessary to remind some anatomic features of the first three ribs. The first rib doesn't necessarily possess a costosternal cartilage - it could be related to manubrium sterni via a pseudoarticulation. The costosternal cartilages and ligaments of ribs II and III are much narrower and shorter than those of the other ribs. The lower a rib is situated in the thorax, the larger its cartilage in the frontal thorax is. The relatively shorter cartilages of the upper ribs reduce their mobility. Thus in case of a costotransversal blockage in the area a much greater overload would be generated in ventral direction. This could explain the predilection for predominant damaging the upper three ribs.

The costal axis of movement (rotation) is of certain importance, too. On the contrary to the lateral movement of the lower ribs, the first four ribs perform a movement towards cranial and forward as the rotation axis passes through the head of the rib (caput costae) and the costotranversal joint being oriented almost parallelly to the frontal plane. This movement is very similar to the swinging of a pail or bucket (5).

\section{Biomechanical considerations about the etiology of Tietze's syndrome}

Having in mind the functional and anatomic characteristics of the first three ribs, the second one bearing the recognition of a 'classic example', it could be accepted that the imbalance of the ribs dorsally leads to disturbances in the frontal thorax, i.e. in the cartilage thus causing a deviation and swallowing of the latter. The limited movement of the rib influences on the movement of rising and descending of the whole frontal thorax. So, a disturbed rib movement pro- 
vokes an irritation ventrally that eventually hinders the thoracic movement in the upper sternal region which participates in the physiological act of breathing.

These changes lead to turning, twisting and other types of stress movements of the costosternal cartilages, especially, of the short and thick cartilage of rib II. This mechanism could be the reason for a painful, aseptic swallowing of the rib cartilage, just the same as the Tietze's syndrome.

\section{Biomechanics and manual findings}

Ribs I, II and III are in extension alongside with a side bending and rotation. The transverse process of vertebra II at the concave side of the scoliosis moves ventrally thus displacing the connected rib II. This ventral pushing of rib II causes the swallowing of the frontal side of the cartilage. If the movement of rib II alone is limited while the other ones are functional, then, inevitably, an additional burdening of the costosternal cartilage of rib II occurs.

\section{Therapy}

An ordinary manual manipulation doesn't cope with Tietze's syndrome for a long time. It leads to a partial symptomatic improvement, however, relapses are usually to develop. A concurrent treating of the lateral bending in the segment makes the relapses rarer and less worsening. A peculiarity of the extension lies in the basis of the posture model of the patients with Tietze's syndrome. The extension is very rarely found in patients with normal posture. Usually, a trauma, false posture or another factor is needed to drive the vertebra I, II and III in dysfunction and the symptoms to start. That is why a prophylaxis of the "extension model' is crucial in the syndrome treatment. An improvement seems more probable if alongside with the extension the lateral bending is treated.

\section{REFERENCES}

1. Aspegren, D, T. Hyde, M. Miller. Conservative treatment of a female collegiate volleyball player with costochondritis.- J. Manipulative Physiol. Ther., 30, 2007, No 4, 321-325.

2. Calabro, J. J., H. Jeghers, K. A. Miller, R. D. Gordon. Classification of anterior chest wall syndromes.- JAMA, 243, 1980, No 14, 1420-1421.
3. Eder, M., H. Tilcher. Schmerztherapie der Wirbelsдule. Stuttgart, Hippokrates, 1991.

4. Fam, A. G., H. A. Smythe. Musculoskeletal chest wall pain.- Can. Med. Assoc. J., 133, 1985, No 5, 379-389.

5. Gijsberg, E., S. F. Knaap. Clinical presentation and chiropractic treatment of Tietze syndrome.- $J$. Chiropr. Med., 10, 2011, No 1, 60-63.

6. Ikehira, H., M. Kinjo, Y. Nagase, H. Ito. Acute pan-costochondritis demonstrated by gallium scintigraphy.- Br. J. Radiol., 72, 1999, No 854, 210-211.

7. Kloth, J. K., M. A. Weber. Weichteilschwellung des Sternoklavikulargelenks.Der Radiologe, 52, 2012, No 5, 459-462.

8. Lauper, M. Thoraxschmerzen aus manualmedizinischer Sicht. SAMM 49. Jahreskongress. Interlaken, 28.XI.2008.

9. Patriquin, D. A. Zur mechanischen Дtiologie des Tietze-Syndroms.- Man. Med., 25, 1987, No 1, 14-17.

10. Rabey, M. I. Costochondritis: are the symptoms and signs due to neurogenic inflammation? Two cases that responded to manual therapy directed towards posterior spinal structures.- Man. Ther., 13, 2008, No $1,82-86$

11. Sain, A. K. Bone scan in Tietze's syndrome.- Clin. Nucl. Med., 3, 1978, No 12, 470-471.

12. Stochkendahl, M. J., H. W. Christensen. Chest pain in focal musculoskeletal disorders.- Med. Clin. North Am., 94, 2010, No 2, 259-273.

13. Tietze, A. Über eine eigenartige Häufung von Fällen mit Dystrophie der Rippenknorpel.- Klin. Wochenschr., 58, 1921, 829-831.

14. Todorov, T. Manual medicine - diagnostics and therapy. Varna, Chernorizets Hrabar, 2005 (in Bulgarian).

15. van Schalkwyk, A. J., J. A. van Wingerden. A variant of Tietze's syndrome occurring after reconstructive breast surgery.- Aesth. Plast. Surg., 22, 1998, No 6, 430-432.

16. Volterrani, L., M. A. Mazzei, N. Giordano, R. Nuti, M. Galeazzi, A. Fioravanti. Magnetic resonance imaging (MRI) in Tietze's syndrome which, to our knowledge, has not previously been reported in the literature.- Clin. Exp. Rheumatol., 26, 2008, No 5, 848-853. 